\title{
A NON-COPRIME HALL-HIGMAN REDUCTION THEOREM
}

\author{
YAN-MING WANG \\ (Received 20 February 1991; revised 5 July 1991)
}

Communicated by H. Lausch

\begin{abstract}
In a well-known paper, Hall and Higman proved the reduction theorem on a coprime order operator group acting on a finite group. This theorem plays an important role in local analysis of finite group theory. In this paper, we generalize the Hall-Higman reduction theorem by dropping the restrictive hypothesis $(|G|,|H|)=1$ and determine the detailed structure of $G$ completely.
\end{abstract}

1991 Mathematics subject classification (Amer. Math. Soc.): 20D10, $20 \mathrm{D} 15$.

\section{Introduction}

It is useful to consider the following critical case when we consider a group acting on another group. Let $G$ be a finite group, and $H$ an operator group of $G$.

HYPOTHESIS ( $\left.{ }^{*}\right) . H$ acts nontrivially on $G$ but acts trivially on every proper $H$ invariant subgroup of $G$.

Our purpose is to determine the structure of a group $G$ which satisfies hypothesis $\left({ }^{*}\right)$. In [2], Hall and Higman have considered this question when $(|G|,|H|)=1$. They proved the following famous reduction theorem.

REDUCTION THEOREM. Suppose that $(G, H)$ satisfies hypothesis $\left(^{*}\right)$ with $(|G|,|H|)=1$. Then $G$ is a special $p$-group.

The hypothesis $(|G|,|H|)=1$ here is very restrictive. For example, the very important case of a subgroup $H$ of $G$ acting on $G$ by conjugation cannot satisfy

(C) 1994 Australian Mathematical Society 0263-6115/94 $\$$ A2.00+0.00

This research was supported by NSF of China 
this coprime order hypothesis. In this paper, we drop this hypothesis and obtain the detailed structure of $G$. We also give some applications and examples.

The main results are as follows:

MAIN THEOREM. Suppose that $(G, H)$ satisfies hypothesis $\left({ }^{*}\right)$. Then, there is a unique maximal $H$-invariant subgroup $C$ of $G$. Moreover, $C=C_{G}(H)=N_{G}(H)$ is a normal abelian subgroup of $G$ and $[G, H] C \leq C_{G}(C)$. Furthermore, $G$ must satisfy one of the following conditions:

(I) $[G, H] \neq G$. Then $|G / C|=p, H / C_{H}(G)$ is isomorphic to a subgroup of $C$ and $H^{\prime}$ acts trivially on $G$. Furthermore, $F(G) \neq C$ if and only if $G$ is a p-group.

(II) $[G, H]=G$. Then $C \leq Z(G)$. Furthermore,

(1) Assume $R_{S}(G) \neq G$. Then $G$ is a p-group in $\mathscr{A}_{e} \cap \mathscr{A}_{e} \mathscr{A A}_{\text {with class } \leq 2 . H}$ acts trivially on $\Phi(G)$ and irreducibly on $G / Z(G), G^{\prime}$ is an elementary $p$-subgroup. If $p \neq 2$, the $x^{p}=1$ for every element $x$ of $G$.

(2) Assume $R_{S}(G)=G$. Then $C=Z(G)=F(G)=\Phi(G)$. Moreover $G / Z(G)=G_{1} \times \ldots \times G_{k}$ is a direct product of isomorphic nonabelian simple groups. For every $i \in\{1, \ldots, k\}$, there exists $H_{i} \leq H$ such that $\left|H: H_{i}\right|=k$, $G_{i} \cong \operatorname{Inn}\left(G_{i}\right) \leq H_{i} / C_{H_{i}}\left(G_{i}\right) \stackrel{\sim}{\leq} \operatorname{Aut}\left(G_{i}\right)$. (Hence $H$ is nonsolvable).

Our notation follows that of [4]. All the groups in this paper are finite. $p$ always denotes a prime. $\mathscr{A}$ denotes the class of abelian groups while $\mathscr{A}_{e}$ denotes the class of elementary abelian groups. $\mathscr{C}_{p}$ denotes the class of $\left\{1, C_{p}\right\}$, where $C_{p}$ is the group of order $p . R_{S}(G)=\cap\{N \mid N \unlhd G$ such that $G / N$ is solvable $\}$.

$[G] H$ expresses the semidirect product of the group $G$ and $H$ where $G \unlhd[G] H$.

\section{Preliminaries}

LEMMA 2.1. Let $M$ be a subgroup of $G$. Suppose that $M \neq 1$ and $N_{G}(P) \leq M$ for every nontrivial $p$-subgroup of $M$ and $p \in \pi(M)$. Then $M$ is a Hall subgroup of $G$ and $G$ is a Frobenius group with Frobenius complement $M$.

ProOF. Let $p \in \pi(M)$ and $P \in \operatorname{Syl}_{p}(M)$. Since $N_{G}(P) \leq M$, we have $P \in$ $\operatorname{Syl}_{p}(G)$. Hence $M$ is a Hall subgroup of $G$. For every $x \in G$ with $M \cap M^{x} \neq 1$, there exists $p|| M \cap M^{x} \mid$. Let $1 \neq P \in \operatorname{Syl}_{p}\left(M \cap M^{x}\right)$. We have $N_{G}(P) \leq M$ and $M^{x}$ and so $P \in \operatorname{Syl}_{p}(G) \cap \operatorname{Syl}_{p}(M) \cap \operatorname{Syl}_{p}\left(M^{x}\right)$. Since $P$ and $P^{x} \in \operatorname{Syl}_{p}(M)$, there exists $m \in M$ such that $P^{m}=P^{x^{-1}}$ by Sylow's theorem. It follows that $x \in M N_{G}(P) \leq M$. Thus $M \cap M^{x}=1$, for every $x \in G-M$. This yields that $G$ is a Frobenius group with Frobenius complement $M$.

LEMMA 2.2. Let $N \leq Z(G), N \cap \Phi(G)=1$. Then $G=G_{1} \times N$ for a subgroup $G_{1}$ of $G$. 
PRoof. Suppose $N \neq 1$. Let $G_{1}=\min \{M \mid N M=G\}$. We assert that $N \cap G_{1}=$ 1. In fact, if $N \cap G_{1} \neq 1$, then there is a maximal subgroup $M$ of $G$ such that $M \nsucceq N \cap G_{1}$ and $\left(N \cap G_{1}\right) M=G$. Thus $M \cap G_{1}<G_{1}$ since $G_{1}<G$, where $G=N G_{1}=N\left(G_{1} \cap\left(\left(N \cap G_{1}\right) M\right)=N\left(N \cap G_{1}\right)\left(M \cap G_{1}\right)=N\left(M \cap G_{1}\right)\right.$, contrary to the choice of $G$. Hence $G=G_{1} \times N$.

LEMMA 2.3. Let $G=G_{1} \times S=G_{2} \times S$, where $S$ is a solvable group and $G_{1}$ is a direct product of some nonabelian simple groups. Then $G_{1}=G_{2}$.

PROOF. $G_{1} \cong G / S \cong G_{2}$. Let $G=G_{11} \times \ldots \times G_{1 k}$, where $G_{1 i}$ is a nonabelian simple group for every $i \in\{1,2, \ldots, k\}$. Since $G_{11} \leq G_{2} \times S$, for each $g_{11} \in G_{11}$, we can uniquely express $g_{11}$ as $g_{11}=g_{2} s$ where $g_{2} \in G_{2}, s \in S$. Set $\sigma: g_{11} \rightarrow s$. Then $\sigma$ is a homomorphism from $G_{11}$ into $S$, since $g_{11}^{1} s^{1} g_{11}^{2} s^{2}=g_{11}^{1} g_{11}^{2} s^{1} s^{2}$. Now $G_{11}$ is nonabelian simple and $S$ is solvable. Hence $\operatorname{Ker} \sigma \neq 1, \operatorname{Ker} \sigma=G$ and so $G_{11} \leq G_{2}$. Similarly, $G_{1 i} \leq G_{2}$ for every $i \in\{1,2, \ldots, k\}$ and finally $G_{1}=G_{2}$.

We say that a group $H$ acts irreducibly on a group $G$ provided that $G$ has no nontrivial proper $H$-invariant subgroup.

LEMMA 2.4. Suppose that a solvable group $H$ acts faithfully and irreducibly on a finite group $G$. Then $G$ is an elementary abelian p-group.

PROOF. The result is trivial if $H=1$. Consider $H \neq 1$. Since $H$ is solvable, there exists a minimal normal $q$-subgroup $Q$ of $H$ such that $C_{G}(Q)$ is $H$-invariant. Irreducibility implies that $C_{G}(Q)=1$. By the orbit formula, $|G|=\left|C_{G}(Q)\right|+k q \equiv$ $1(\bmod q)$, so $G$ is a $q^{\prime}$. group. By Glauberman's theorem [4, Theorem 7.5] there exists $P \in \operatorname{Syl}_{p}(G)$ such that $P$ is $Q$-invariant for every $p \in \pi(G)$. The same theorem yields that the $Q$-invariant Sylow $p$-subgroup is unique since $C_{G}(Q)=1$. Since $Q \unlhd H$, we have $\left(P^{h}\right)^{Q}=\left(P^{Q}\right)^{h}=P^{h}$. Hence $P^{h}=P$ by uniqueness and so $P$ is $H$-invariant. Again, irreducibility yields that $G=P$ and $\Phi(P)=1$, that is , $G$ is an elementary abelian $p$-group.

\section{Proof of the main theorem}

LEMMA 3.1. Suppose that $(G, H)$ satisfies hypothesis $\left(^{*}\right)$. Then:

(1) $G /[G, H]$ is a cyclic p-group or the identity group.

(2) The unique maximal $H$-invariant subgroup of $G$ is $C=C_{G}(H)=N_{G}(H)$ and $C$ is a normal subgroup of $G$.

(3) $[G, H] \leq C_{G}(N)$ for every proper $H$-invariant subgroup $N$ of $G$. 
Proof. (1) The conclusion is obvious if $G=[G, H]$. Suppose $[G, H]<G$. Since $H$ acts trivially on $G /[G, H]$ but nontrivially on $G$, there exists $x \in G$ such that $[x, H] \neq 1$ and $\langle x\rangle[G, H]$ is an $H$-invariant subgroup of $G$. Hypothesis $\left(^{*}\right)$ yields that $G=\langle x\rangle[G, H]$, that is, $G /[G, H]$ is cyclic. Let $G /[G, H]=P_{1} /[G, H] \times \ldots \times$ $P_{k} /[G, H]$ be the direct product of Sylow subgroups, where each $P_{i}$ is $H$-invariant. We have $k=1$ and $G /[G, H]$ is a cyclic $p$-group by hypothesis $\left(^{*}\right)$.

(2) It is clear that every proper $H$-invariant subgroup of $G$ is contained in $C=$ $C_{G}(H)$. Therefore $C$ is the unique maximal $H$-invariant subgroup of $G$. We claim that $N_{G}(H)=C$. In fact, if $N_{G}(H)>C$, we have $N_{G}(H)=G$ since $N_{G}(H)$ is $H$-invariant. It is easy to see that $[G, H]=1$ in this case, contrary to the hypothesis. Our next goal is to show that $C \unlhd G$ by induction on $|G|$. If $C=1$, there is nothing to prove. We consider $C \neq 1$. Set $\operatorname{Core}(C)=\bigcap_{x \in G} C^{x}$. Suppose Core $(C)=1$. Then there is no nontrivial normal subgroup of $G$ contained in $C$. For each $p \in \pi(C)$, let $1 \neq P$ be a $p$-subgroup of $C$. Since $N_{G}(P)$ is $H$-invariant and $N_{G}(P) \neq G$, we have $N_{G}(P) \leq C$. Now $G=K[C]$ by Lemma 2.1 , where $K$ is the Frobenius core and so is $H$-invariant. Since $C \neq 1$, it follows that $K \neq G$ and hence $K \leq C$. Thus $G \leq C \neq G$, a contradiction. Thus we have $\operatorname{Core}(C) \neq 1$. Consider $H$ acting on $\bar{G}=G / \operatorname{Core}(C)$. Suppose $H$ acts nontrivially on $\bar{G}$. Then $(\bar{G}, H)$ satisfies hypothesis $\left(^{*}\right)$ and the unique maximal $H$-invariant subgroup of $\bar{G}$, $C_{\bar{G}}(H)=L / \operatorname{Core}(C)$ is normal in $\bar{G}$ by induction. It is clear that $L \geq C$ and $L \neq G$. We conclude that $C=L \unlhd G$. If $H$ acts trivially on $G$, then $[G, H] \leq \operatorname{Core}(C) \leq C$ and $G / \operatorname{Core}(C)$ is cyclic, hence $C \unlhd G$. This completes the proof of (2).

(3) Since $[C, H]=1$ and $C \unlhd G$, we have $[C, H, G]=1=[G, C, H]$. Thus we have $[G, H] \leq C_{G}(C)$. Hence $[G, H] \leq G_{G}(N)$ for every proper $H$-invariant subgroup $N$ of $G$ by (2).

In the following, $C$ always denotes $C_{G}(H)$.

THEOREM 3.1. Suppose that $(G, H)$ satisfies hypothesis $\left(^{*}\right)$ with $[G, H] \neq G$. Then $|G / C|=p$ and $C$ is an abelian group. Moreover $H / C_{H}(G)$ is isomorphic to a subgroup of $C$, and $H^{\prime}$ acts trivially on $G$.

Proof. Since $[G, H]<G,[G, H]$ is normal and $H$-invariant in $G$, and $[G, H] \leq$ $C$ by Lemma 3.1 (3). Since $H$ acts trivially on $G / C$ and $C$ is a maximal $H$ invariant subgroup of $G$, we find $G / C$ has no nontrivial proper subgroup, and so $|G / C|=p$ for some prime $p$. Since $H$ acts trivially on both $G / C$ and $C$ but nontrivially on $G$, there is some $a \in G$ such that $[a, H] \neq 1$ and $\langle a\rangle C=G$. For each $h \in H$, there is an unique $c_{h} \in C$ such that $a^{h}=c_{h} a$. Set $\sigma: h \rightarrow c_{h}$. Then $\sigma$ is a homomorphism from $H$ to $C$. In fact, if $a^{h_{1}}=c_{h_{1}} a, a^{h_{2}}=c_{h_{2}} a$, then $a^{h_{1} h_{2}}=\left(c_{h_{1}} a\right)^{h_{2}}=c_{h_{1}} c_{h_{2}} a$. Since $\operatorname{Ker} \sigma=C_{H}(a)=C_{H}\langle a, C\rangle=C_{H}(G)$, we have that $H / C_{H}(G)$ is isomorphic to a subgroup of $C$. It remains to prove that $C$ is abelian. 
For every $x \in C, C \unlhd G$ yields that $x^{a} \in C$. Since $H$ acts trivially on $C$, we get $x^{a}=\left(x^{a}\right)^{h}=\left(a^{-1} x a\right)^{h}=\left(a^{h}\right)^{-1} x^{h} a^{h}=a^{-1} c_{h}^{-1} x c_{h} a$ for every $h \in H$. Thus $x c_{h}=c_{h} x, c_{h} \in Z(C), a^{h}=c_{h} a \in Z(C)\langle a\rangle$. Since $Z(C) \operatorname{char} C \unlhd G, Z(C)\langle a\rangle$ is a subgroup of $G$. Hence $G_{1}=\left\langle a^{h} \mid h \in H\right\rangle \leq Z(C)\langle a\rangle$. It is clear that $a \in G_{1}, G_{1}$ is $H$-invariant and $H$ acts nontrivially on $G_{1}$. So $G=G_{1} \leq Z(C)\langle a\rangle$ by hypothesis $\left({ }^{*}\right)$. It follows that $C / Z(C)$ is cyclic and $C$ is abelian.

REMARK 1. By Lemma 3.1(3) and Theorem 3.1, we have that $C$ is abelian in all cases (independently of whether $[G, H]=G$ or not).

THEOREM 3.2. Suppose that $(G, H)$ satisfies hypothesis $\left({ }^{*}\right)$ with $[G, H]=G$. Then

(1) every proper $H$-invariant subgroup of $G$ is contained in $Z(G)$;

(2) furthermore, if $R_{S}(G) \neq G$, then we have the following:

(a) $G$ is a p-group in $\mathscr{A}_{e} \cap \mathscr{A}_{e} \mathscr{A}$ with class at most two.

(b) $G^{\prime} \leq \Phi(G) \leq Z(G), G^{\prime}$ is an elementary abelian p-group. $H$ acts trivially on $\Phi(G)$ and irreducibly on $G / Z(G)$.

(c) If $p \neq 2$, then $x^{p}=1$ for every $x \in G$.

PROOF. (1) For each proper $H$-invariant subgroup $N$ of $G$, we have $G=$ $[G, H] \leq C_{G}(N)$ by Lemma 3.1 (3). Thus $N \leq Z(G)$.

(2) If $R_{S}(G) \neq G$, then $R_{S}(G) \leq Z(G)$ by (1). So $G / Z(G)$ is solvable and $G^{\prime}<G$, and $G^{\prime} \leq Z(G)$ by (1). Therefore $G / Z(G)$ is abelian and $G$ is nilpotent. It is easy to show that $G$ is a $p$-group by hypothesis $\left({ }^{*}\right)$.

Proof of (b). It is clear that $G^{\prime} \leq \Phi(G) \leq Z(G)$. $H$ acts trivially on $\Phi(G)$ since $\Phi(G) \neq G$. By (1) and $\Phi(G) \leq Z(G)$ we conclude that $G / Z(G)$ is elementary abelian and $H$ acts irreducibly on $G / Z(G)$. For $x, y \in G$, we have $y^{p} \in \Phi(G) \leq$ $Z(G)$ and $[x, y] \in Z(G)$. Thus $1=\left[x, y^{p}\right]=[x, y]^{p}$ and so $G^{\prime}$ is an elementary abelian $p$-group.

Proof of (a). $G / G^{\prime} \in \mathscr{A}, G^{\prime} \in \mathscr{A}_{e}$ by (b), and $G / Z(G) \in \mathscr{A}_{e}$. Hence $G \in$ $\mathscr{A}_{e} \cap \mathscr{A}_{e} \mathscr{A}$ and the class of $G$ is at most 2 .

Proof of (c). If $p \neq 2$, then $p \mid p(p-1) / 2$. Since $H$ acts trivially on $G^{p} G^{\prime},\left(G^{\prime}\right)^{p}=$ 1 and $G^{\prime} \leq Z(G)$, and we have $[x, h]^{p}=\left(x^{-1} x^{h}\right)^{p}=\left(x^{p}\right)^{-1}\left(x^{P}\right)^{h}\left[x^{-1}, x^{h}\right]^{p(p-1) / 2}=$ 1. The conclusion follows from that $[G, H]=G$ and $(x y)^{p}=x^{p} y^{p}[x, y]^{p(p-1) / 2}$.

\section{LEMMA 3.2.}

(1) Suppose that group $H$ acts faithfully and irreducibly on a nonabelian simple group $G$. Then $G \cong \operatorname{Inn}(G) \leq H \leq \operatorname{Aut}(G)$.

(2) Suppose that a group $H$ acts faithfully and irreducibly on a nonsolvable group G. Then $G=G_{1} \times \ldots \times G_{k}$ is a direct product of isomorphic nonabelian simple 
groups, and for each $i \in\{1, \ldots, k\}$, there is $H_{i} \leq H$ with $\left|H: H_{i}\right|=k$, such that

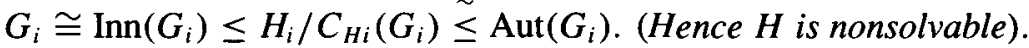

Proof. (1) Consider $L=[G] H$. Then $C_{L}(G) \unlhd L$. It is obvious that $\operatorname{Inn}(G) \cong$ $G$ and $H=N_{H}(G) / C_{H}(G) \leq \operatorname{Aut}(G)$. We only need to prove $\operatorname{Inn}(G) \leq H$.

(a) We claim $C_{L}(H) \neq 1$.

If this is false, then $H \cong L / G \leq \operatorname{Aut}(G) / \operatorname{Inn}(G)$ since $L=N_{L}(G) / C_{L}(G)$ $\leq \operatorname{Aut}(G)$. Now Aut $(G)$ is solvable by [1, Theorem 4.239] and so $H$ is solvable. By Lemma $2.4, G$ is a $p$-group, which contradicts the fact that $G$ is nonabelian simple.

(b) $G=\left\{g \mid g \in G, \exists h \in H\right.$, such that $\left.g h \in C_{L}(G)\right\}$.

Set $G_{1}=\left\{g \mid g \in G, \exists h \in H\right.$, such that $\left.g h \in C_{L}(G)\right\}$. Since $G_{H}(G)=1$ and $C_{L}(G) \neq 1$, we have $1 \in G_{1}$ and $G_{1} \neq\{1\}$. Let $g_{1}, g_{2} \in G_{1}, h_{1}, h_{2} \in H$ be such that $g_{i} h_{i} \in C_{L}(G), i=1,2$. Then $h_{1}=g_{1}^{-1}\left(g_{1} h_{1}\right)=g_{1} h_{1} g_{1}^{-1}, g_{1} h_{1}=h_{1} g_{1}$, $g_{1}^{-1} h_{1}^{-1}=\left(g_{1} h_{1}\right)^{-1} \in C_{L}(G)$, so $g_{1}^{-1} \in G_{1}$. Moreover $\left(g_{2} g_{1}\right)\left(h_{1} h_{2}\right)=g_{2}\left(g_{1} h_{1}\right) h_{2}=$ $g_{1} h_{1} g_{2} h_{2} \in C_{L}(G)$. Thus $G_{1}$ is a subgroup of $G$. For each $h \in H, g_{1}^{h} h_{1}^{h}=\left(g_{1} h_{1}\right)^{h} \in$ $C_{L}(G)^{h}=C_{L}(G)$. Thus $g_{1}^{h} \in G_{1}$. It follows that $1 \neq G_{1}$ is an $H$-invariant subgroup of $G$ and so $G=G_{1}$.

(c) There is an injective map from $G$ to $H$. In fact, for each $g \in G$, there is $h_{g} \in H$ such that $g h_{g} \in C_{L}(G)$. If $g h_{g}=g h_{g^{\prime}}$, then $h_{g}^{-1} h_{g}^{\prime}=\left(g h_{g}\right)^{-1} g h_{g_{g}^{\prime}} \in$ $C_{L}(G) \cap H=C_{H}(G)=1$, so $h_{g}=h_{g^{\prime}}$. Thus $h_{g}$ is uniquely determined by $g$. Let $\sigma: g \rightarrow h_{g}^{-1}$. We assert that $\sigma$ is an injective homomorphism from $G$ to $H$. In fact, suppose $g_{1} h_{g_{1}}, g_{2} h_{g_{2}} \in C_{L}(G)$. Then $g_{1} g_{2} h_{g_{2}} h_{g_{1}}=g_{2} h_{g_{2}} g_{1} h_{g_{1}} \in C_{L}(G)$. Thus $\sigma\left(g_{1} g_{2}\right)=\left(h_{g_{2}} h_{g_{1}}\right)^{-1}=h_{g_{1}}^{-1} h_{g_{2}}^{-1}=\sigma\left(g_{1}\right) \sigma\left(g_{2}\right)$, so $\sigma$ is a homomorphism. Now $\operatorname{Ker} \sigma \unlhd G$. If $\operatorname{Ker} \sigma=G$, then $h_{g}=1$, for every $g \in G$ and so $G \leq C_{L}(G)$. Thus $G$ is abelian, a contradiction. Therefore $\operatorname{Ker} \sigma=1$ since $G$ is simple.

For each $I_{g} \in \operatorname{Inn}(G), x \in G, x^{g h_{g}}=x, x^{g}=x^{h_{g}^{-1}}$. Hence $H$ contains every $I_{g}$. It follows that $G \cong \operatorname{Inn}(G) \leq H \leq \operatorname{Aut}(G)$.

Proof of (2). $H$ acts irreducibly on $G$, so $G$ is characteristically simple. Hence $G=G_{1} \times \ldots \times G_{k}$ is a direct product of isomorphic nonabelian simple groups. Let $H_{1}=N_{H}\left(G_{1}\right)$. Let $H=H_{1}+H_{1} a_{2}+\ldots+H_{1} a_{n},\left\{1=a_{1}, a_{2}, \ldots, a_{n}\right\}$ be the transversal of $H_{1}$ in $H$. Since $G_{1}$ is a minimal normal subgroup of $G, G_{1}^{a_{i}}=G_{1}^{a_{j}}$, if and only if $a_{i}=a_{j}$. Now $1 \neq\left\langle G_{1}^{h}: h \in H\right\rangle=G_{1} \times G_{1}^{a_{2}} \times \ldots \times G_{1}^{a_{n}}=G$ since $\left\langle G_{1}^{h}: h \in H\right\rangle$ is an $H$-invariant subgroup of $G$. It is clear that $k=n$, hence $\mid H$ : $H_{1} \mid=k$. If $1 \neq K_{1}<G$ and $K_{1}$ is $H_{1}$-invariant, then $1 \neq K=K_{1} \times \ldots \times K_{1}^{a_{k}}<G$ and $K$ is $H$-invariant, contrary to irreducibility. It follows that $H_{1}$ acts irreducibly on $G$. Hence $G_{i} \cong \operatorname{Inn}\left(G_{i}\right) \leq H_{i} / C_{H i}\left(G_{i}\right) \stackrel{\sim}{\leq} \operatorname{Aut}\left(G_{i}\right)$ by (1). The same argument for $i$ implies the conclusion.

THEOREM 3.3. Suppose that $(G, H)$ satisfies the hypothesis $\left({ }^{*}\right)$ with $R_{S}(G)=G$. Then $G=[G, H]$ and $C=C_{G}(H)=Z(G)=F(G)=\Phi(G)$. $G$ is a perfect 
quasinilpotent group, and $\bar{G}=G / Z(G)=G_{1} \times \ldots \times G_{k}$ is a direct product of isomorphic nonabelian simple groups. For each $i \in\{1, \ldots, k\}$, there is $H_{i} \leq H$ with $\left|H: H_{i}\right|=k$, such that $G_{i} \cong \operatorname{Inn}\left(G_{i}\right) \leq H_{i} / C_{H_{i}}\left(G_{i}\right) \leq \operatorname{Aut}\left(G_{i}\right)$. (Hence $H$ is nonsolvable).

PRoOF. $G=[G, H]$ follows from Theorem 3.1. Since $R_{S}(G)=G, G=G^{\prime}$ and $G$ is perfect. Thus $F(G) \neq G \neq Z(G)$, hence $F(G)=C=Z(G)$ by Theorem 3.2 (1) and Lemma 3.1. Hence $\bar{G}=G / Z(G)$ has no nontrivial proper $H$-invariant subgroup, so $\bar{G}=G_{1} \times \ldots \times G_{k}$ has the properties mentioned in Lemma 3.2. $G$ is perfect nilpotent by [3, X Section 13]. There is only $\Phi(G)=Z(G)$ left to prove. We prove this by induction on $|G|$.

Firstly, we consider $\Phi(G)=1$. If $Z(G) \neq 1$, then $G=Z(G) \times G_{1}$ by Lemma 1.2. Since $G_{1} \cong G / Z(G)$ is a direct product of isomorphic nonabelian simple groups, $Z(G) \times G_{1}=G=G^{h}=Z(G)^{h} \times G_{1}^{h}, \forall h \in H$. Hence $G_{1}=G_{1}^{h}$ by Lemma 1.3. thus $G_{1} \neq G$ and $G_{1}$ is $H$-invariant. This yields that $G_{1} \leq Z(G)$ and $G \leq Z(G) \neq G$, a contradiction. We conclude that $Z(G)=C=1$ if $\Phi(G)=1$.

Now, consider $\Phi(G) \neq 1$. Consider $H$ acting on $G / \Phi(G)$. If $H$ acts trivially on $G / \Phi(G)$, then $G=[G, H] \leq \Phi(G)$, a contradiction. Hence $(G / \Phi(G), H)$ satisfies hypothesis $\left({ }^{*}\right)$ and $R_{S}(G / \Phi(G))=G / \Phi(G), \Phi(G / \Phi(G))=1$. The proceeding argument yields that $1 \neq Z(G / \Phi(G)) \geq Z(G) / \Phi(G)$ and so $\Phi(G)=z(G)=C$.

The main Theorem follows from Theorem 3.1, Theorem 3.2 and Theorem 3.3.

\section{Applications and examples}

COROLLARY 4.1. Let $G$ be a solvable group. Let $H$ be an operator group of $G$.

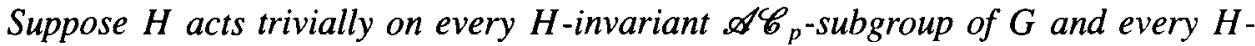
invariant p-subgroup of $G$ which lies in $\mathscr{A}_{e} \cap \mathscr{A}_{e} \mathscr{A}$ with class at most two. Then $H$ acts trivially on $G$.

PROOF. Assume that the conclusion is false and let $G$ be a counterexample of minimal order. Since the hypotheses in Corollary 4.1 are inherited for $H$-invariant subgroups, by the choice of $G, H$ acts trivially on every proper $H$-invariant subgroup of $G$. Thus $(G, H)$ satisfies hypothesis $\left({ }^{*}\right)$. Since $G$ is solvable, by Theorem 3.1 and Theorem $3.2, G \in \mathscr{A C}_{p}$ or $G$ is a $p$-group in $\mathscr{A} \mathscr{A}_{e} \cap \mathscr{A}_{e} \mathscr{A}$ with class at most two. Thus $H$ acts trivially on $G$, contrary to our choice. This shows that there is no counterexample and the corollary is proved.

COROLLARY 4.2. Suppose that $H$ is a solvable operator group of $G$ and $H$ acts nontrivially on $G$ but acts trivially on every proper $H$-invariant $\mathscr{A}_{\mathscr{C}_{p}}$-subgroup of 
$G$ and every proper $H$-invariant $p$-subgroup with class at most two which lies in $\mathscr{A}_{e} \cap \mathscr{A}_{e} \mathscr{A}$. Then $G$ is solvable.

PROOF. Assume that the conclusion is false and let $G$ be a counterexample of minimal order. We assert that $(G, H)$ satisfies hypothesis $\left({ }^{*}\right)$.

In fact, $H$ acts nontrivially on $G$. Suppose $H$ acts nontrivially on a proper $H$ invariant subgroup $G_{1}$ of $G$. Then $G_{1}$ satisfies the assumption of Corollary 4.2; thus $G_{1}$ is solvable by the choice of $G$. Hence $(G, H)$ satisfies hypothesis $\left(^{*}\right)$. By Theorem 3.2, $C=C_{G}(H) \unlhd G$ and $C$ is abelian. Consider $H$ acting on $G / C$.

Assume $C \neq 1$. If $H$ acts trivially on $G / C$, then $G / C$ is cyclic by Lemma 3.1 and so $G$ is solvable, a contradiction. If $H$ acts nontrivially on $G / C$, then $(G / C, H)$ satisfies hypothesis $\left(^{*}\right)$ and so satisfies the hypothesis of Corollary 4.2. The choice of $G$ yields that $G / C$ is solvable and hence so is $G$, contrary to our choice.

Therefore $C=1$. Since $(G, H)$ satisfies hypothesis $\left({ }^{*}\right)$, Lemma 3.1 yields that every proper $H$-invariant subgroup of $G$ is contained in $C=1$. Hence $H$ acts irreducibly on $G$. Since $H$ is solvable, by Lemma $2.4, G$ is an elementary abelian $p$-group, contrary to our assumption. This shows that there is no counterexample and the corollary is proved.

We say that a group $G$ is 3-step solvable if $G^{(3)}=1$.

COROLLARY 4.3. Suppose that $A$ is an abelian subgroup of $G$ and suppose $A$ lies in the centre of every 3-step solvable subgroup of $G$ which contains $A$. Then $A$ lies in the centre of $G$.

Proof. Consider $A$ acting on $G$ by conjugation. Assume that the conclusion is false and let $G$ be a counterexample. Now $A \not Z Z(G)$ means that $A$ acts nontrivially on $G$. For every $M \leq G$, where $M$ is $A$-invariant and either $M \in \mathscr{A} \mathscr{C}_{p}$ or $M$ is a $p$-group in $\mathscr{A}_{e} \cap \mathscr{A}_{e} \mathscr{A}$ with class at most two, we have $M^{(2)}=1$ and $M \unlhd M A$, $(M A)^{\prime} \leq M$. Hence $(M A)^{(3)}=1$. By assumption, $A \leq Z(M A)$, that is, $A$ acts trivially on $M$. Corollary 4.2 yields that $G$ is solvable. Now, Corollary 4.1 forces that $A$ acts trivially on $G$, contrary to our assumption. This completes the proof of the theorem.

COROLLARY 4.4. A p-element $x$ of $G$ lies in $Z(G)$ if and only if both of the following hold

(1) there exists $P \in \operatorname{Syl}_{p}(G)$, such that $P \cap\left\{x^{g} \mid g \in G\right\}=x$;

(2) $x$ centralizes every $p^{\prime}$-characteristic subgroup of $M$, where $M$ is a 3-step solvable subgroup of $G$ which contains $x$.

PROOF. Similar to the proof of Corollary 4.3 
REMARK 2.

(1) Corollaries 4.3 and 4.4 are generalisations of the main results in [5].

(2) Suppose $(G, H)$ satisfies hypothesis $\left(^{*}\right)$ with $(|G|,|H|)=1$. Then $G$ is described by case II(1) of the Main Theorem, by Glauberman's theorem. We can easily prove that $G$ is a special $p$-group and obtain the original Hall-Higman theorem.

(3) The tools used in the proof of the applications above are elementary; all of them can be found in [4].

We give an example for each case in the Main Theorem.

(I) $G=A_{4}=\left[B_{4}\right] C_{3}$ where $B_{4}$ is Klein 4-group. Let $H=B_{4}$ with $H$ acting on $G$ by conjugation. Every proper $H$-invariant subgroup of $G$ is contained in $B_{4}$.

(II) (1) Let $G$ be any cyclic group of order $P, p$ an odd prime, $1 \neq H \leq \operatorname{Aut}(G)$.

(II) (2) (i) Let $G$ be a nonabelian characteristic simple group, $H=\operatorname{Aut}(G)$.

(ii) Let $G / Z(G)$ be a nonabelian simple group, $G=H, H$ acting on $G$ by conjugation.

\section{Acknowledgments}

The author deeply thanks Professor Chen Zhong-mu for his kind advice and help. The author wishes to thank the referee for several helpful comments, including a suggestion for Remark 1.

\section{References}

[1] D. Gorenstein, Finite simple groups (Plenum Press, New York, 1982).

[2] P. Hall and G. Higman, 'The $p$-length of $p$-solvable groups and reduction theorems for Burnside's problem', Proc. London Math. Soc. 6 (3) (1956), 1-14.

[3] B. Huppert and N. Blackburn, Finite groups III (Springer, New York, 1967).

[4] H. Kurzweil, Endliche Gruppen (Springer, New York, 1967).

[5] E. Schult, 'Some analogues of Glauberman's Theorem', Proc. Amer. Math. Soc. 17 (1966), 1186 1190.

Mathematics Department

Zhongshan University

Guangzhou 510275

People's Republic of China 